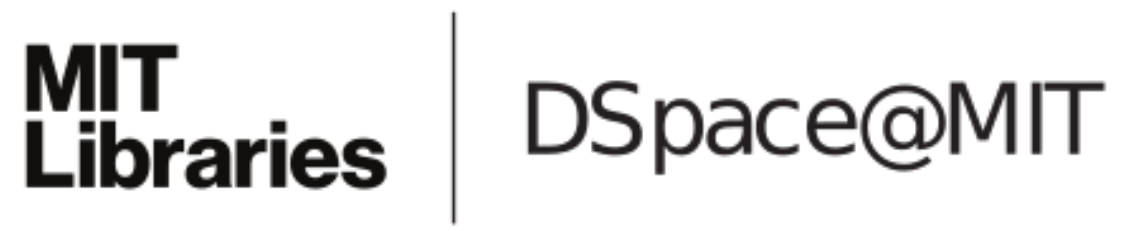

\author{
MIT Open Access Articles
}

\begin{abstract}
Multi-stage optimization of ultrabroadband highenergy optical parametric chirped pulse amplification
\end{abstract}

The MIT Faculty has made this article openly available. Please share how this access benefits you. Your story matters.

Citation: Moses, J. et al. “Multi-stage optimization of ultrabroadband high-energy optical parametric chirped pulse amplification." Lasers and Electro-Optics, 2009 and 2009 Conference on Quantum electronics and Laser Science Conference. CLEO/QELS 2009. Conference on. 2009. 1-2. Print. (C) 2009 Institute of Electrical and Electronics Engineers

As Published: http://ieeexplore.ieee.org/xpls/abs_all.jsp?arnumber=5225449

Publisher: Institute of Electrical and Electronics Engineers

Persistent URL: http://hdl.handle.net/1721.1/67841

Version: Final published version: final published article, as it appeared in a journal, conference proceedings, or other formally published context

Terms of Use: Article is made available in accordance with the publisher's policy and may be subject to US copyright law. Please refer to the publisher's site for terms of use. 


\title{
Multi-Stage Optimization of Ultrabroadband High-Energy Optical Parametric Chirped Pulse Amplification
}

\author{
Jeffrey Moses ${ }^{1}$, Cristian Manzoni ${ }^{2}$, Shu-Wei Huang ${ }^{1}$, Giulio Cerullo ${ }^{2}$, and Franz X. Kärtner ${ }^{1}$ \\ ${ }^{1}$ Department of Electrical Engineering and Computer Science and Research Laboratory of Electronics, \\ Massachusetts Institute of Technology, Cambridge, MA 02139, USA \\ ${ }^{2}$ ULTRAS-INFM-CNR, Dipartimento di Fisica, Politecnico di Milano, Piazza L. da Vinci 32, 20133 Milano, Italy \\ j_moses@mit.edu
}

\begin{abstract}
We present a procedure for simultaneous optimization of efficiency-bandwidth product and superfluorescence noise suppression in ultrabroadband high-energy optical parametric chirped pulse amplification. Gain dependence of parameters makes stage-by-stage signal chirp optimization necessary in multi-stage amplification.

(C) 2009 Optical Society of America

OCIS codes: (320.7110) Ultrafast nonlinear optics; (190.4970) Parametric oscillators and amplifiers
\end{abstract}

Optical parametric chirped pulse amplification (OPCPA) has become valuable not only as a means to push the limits of high peak power pulse generation at wavelengths at which laser amplification has not been developed, but as the only demonstrated technology for producing few-cycle pulses beyond the terawatt peak-power level [1]. Central to the emergence of OPCPA technology is the issue of maintaining ultrabroadband amplification while pushing the limits of efficient energy conversion from pump to signal. Additionally, the OPCPA may combine low seed energy $(<<1 \mathrm{~nJ})$ with high desired gain. Under these conditions, the presence of noise at signal wavelengths due to spontaneous parametric generation cannot be ignored. Such amplified noise or "superfluorescence" may overtake the signal amplification, causing a strong decrease in amplified signal energy and stability, and introduce a temporal pedestal in the compressed pulse which may be detrimental for some applications. Indeed, recent reports of OPCPA in the mid-infrared have found superfluorescence to be a major limiting factor in signal energy scaling $[2,3]$.

In this paper, we employ analytical and numerical analyses, supported by experiment, to investigate the simultaneous optimization of conversion efficiency, signal bandwidth, and signal-to-noise ratio in ultrabroadband, high-energy OPCPA. We find that each of these properties is strongly tied to the ratio of seed and pump pulse durations, and that due to dependence of the optimization problem on the peak amplifier gain, the seed pulse chirp must be optimized independently in each stage of a multi-stage OPCPA system. Additionally, we find that a small reduction in signal bandwidth strongly enhances the signal-to-noise ratio and conversion efficiency. These principles have since been used to build a high-energy, 3-cycle, 2- $\mu$ m amplifier with low noise and good energy stability [4].
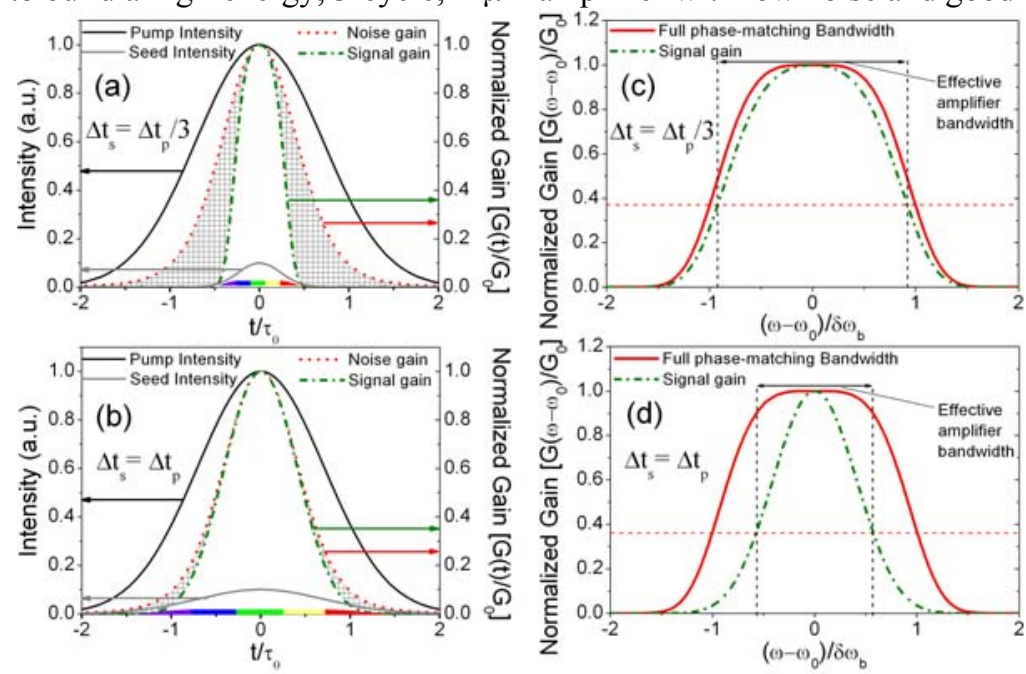

Figure 1. (a,b) Gaussian pump (black, solid) and seed (gray, solid) intensity profiles with corresponding signal gain (green, dot-dashed) and noise gain (red, dotted) profiles for different ratios of seed and pump pulse durations $\left(\Delta \mathrm{t}_{\mathrm{s}}, \Delta \mathrm{t}_{\mathrm{p}}\right)$. The shaded region represents the difference between noise and signal gain. The chirp of the signal pulse is represented by colored bars. (c,d) Corresponding signal gain profiles (green, dot-dashed) in the frequency domain, plotted alongside the full phasematching bandwidth of the amplifier (red, solid).

Figure 1 illustrates the dependence of conversion efficiency, amplifier bandwidth, and signal-to-noise ratio in OPCPA on the seed chirp. The panels on the left plot the signal gain profile, $G=1+(\Gamma / \gamma)^{2} \sinh ^{2}(\gamma \mathrm{L})$, for a chirped seed pulse overlapped with a Gaussian pump pulse with intensity $I_{\mathrm{p}}(\mathrm{t})$, alongside the corresponding noise gain 


\section{CWL2.pdf}

profile; here $\gamma=\left(\Gamma^{2}-(\Delta \mathrm{k} / 2)^{2}\right), \Gamma$ is the nonlinear drive (proportional to the square-root of $I_{\mathrm{p}}$ ), and $\Delta \mathrm{k}$ is the wavevector mismatch. Since noise photons produced by spontaneous parametric generation will be statistically equally distributed in time and frequency, the instantaneous noise gain is determined solely by the local pump intensity, $I_{\mathrm{p}}(\mathrm{t})$, while the instantaneous signal gain is further reduced by the local wave-vector mismatch $\Delta k(t)$. With $\Delta t s=\Delta t p / 3$ (small seed chirp, Fig. 1a) the signal pulse fits within a largely unvarying portion of the pump intensity profile. As a result, there is little clipping of the signal pulse at the wings, and the effective amplifier bandwidth is nearly the full phase-matching bandwidth (Fig. 1c). However, since the signal carrier frequency sweeps quickly in time, so does $\Delta k(t)$, and as a result the gain profile of the signal is much narrower than that of the noise. The narrow signal gain profile means the conversion efficiency will be small, as only a fraction of the pump pulse will be depleted, and the large area between signal and noise gain profiles means the signal-to-noise ratio will strongly degrade after amplification. At $\Delta t s=\Delta t p$ (large seed chirp, Fig. 1b), the slower variation in $\Delta k(t)$ allows the signal gain profile to widen relative to the noise gain profile, resulting in larger conversion efficiency and higher signal-to-noise ratio. On the other hand, since there is more spectral clipping of the signal pulse during amplification, there is a narrower effective amplifier bandwidth. This bandwidth reduction strongly improves the robustness of signal-to-noise ratio of the amplifier; in fact, since the effective amplifier bandwidth covers only the flat, phase-matched central region of the gain bandwidth, $\Delta k$ is essentially zero over the full significant gain region of the pump pulse. Therefore, there is little preferential amplification of the noise.

We have verified the qualitative predictions of Fig. 1 by means of numerical simulations solving the coupled nonlinear equations in a 3-mm long periodically poled stoichiometric lithium tantalate crystal pumped by a 9-ps pulse. In Fig. 2 we show excellent agreement between numerical and experimental results for the measured spectra and efficiencies [3]. We see a monotonic rise in maximum conversion efficiency and a monotonic fall in amplifier bandwidth as seed chirp is increased, as expected from the previous discussion. There is close quantitative agreement in both efficiency-bandwidth product maximum and spectral trend between numerics and experiment. The simulations of signal-to-noise evolution (not shown) verify the predicted increase in signal-to-noise ratio as the seed chirp is increased, which occurs sharply until roughly the same chirp that maximizes efficiency-bandwidth product is reached; from that point on the signal-to-noise ratio flattens out.

Our results further suggest a need for stage-by-stage optimization of the signal chirp. The chirp that maximizes efficiency-bandwidth product and insures robustness of signal-to-noise ratio decreases with increasing gain, due to the increase in gain narrowing. We have found our simulation results to be fit well by an analytic formula for temporal signal gain profile width, $\left(\Delta t_{s} / \Delta t_{p}\right)_{\text {OPт }} \approx \alpha\left(-2 \ln \left[1-1 / \ln \left(4 G_{0}\right)\right]\right)^{1 / 2}$, where $G_{0}$ is the peak signal gain. According to this result, a power amplifier stage with $G_{0}=10^{2}$ will have 1.5 times larger an optimal chirp than a preamplifier stage with $G_{0}=10^{5}$. In order to prevent stage-to-stage buildup of superfluorescence noise while maintaining an ultrabroad bandwidth, the seed chirp should be readjusted at each amplification stage.

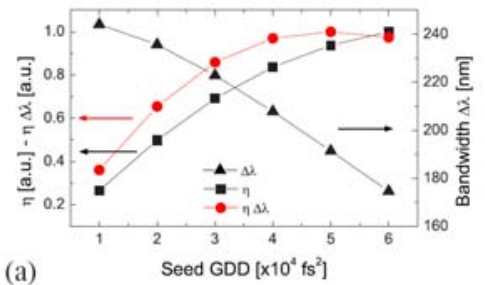

(a)

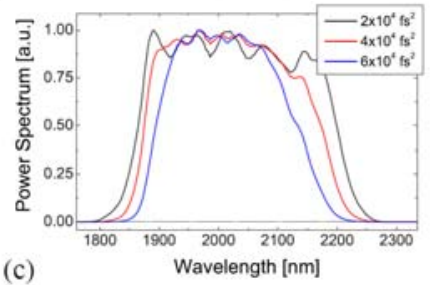

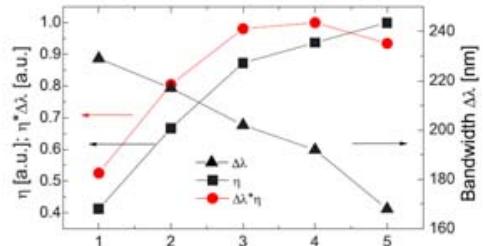

(b)

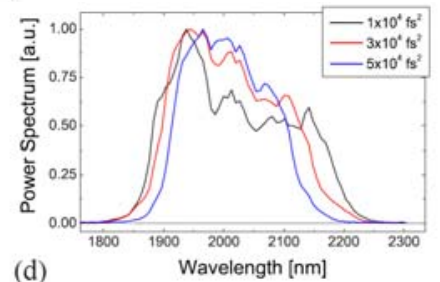

Fig. 2. Comparison between numerical simulations $[(\mathrm{a}),(\mathrm{c})]$ and experiments $[(\mathrm{b}),(\mathrm{d})]$ for a $2-\mu \mathrm{m}$ OPCPA pumped by a 9ps pulse at $1 \mu \mathrm{m}$. (a)-(b) Best conversion efficiencies (squares) and bandwidths (triangles) as a function of seed duration. (c)-(d) Amplified spectra corresponding to highest efficiencies for each of the three given seed durations.

\section{References}

[1] F. Tavella, et al., "Dispersion management for a sub-10-fs, 10 TW optical parametric chirped-pulse amplifier,"Opt. Lett. 32, 2227 (2007).

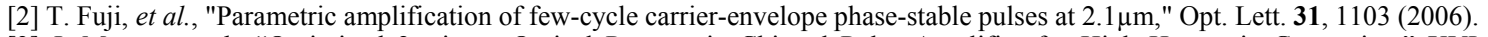

[3] J. Moses, et al., "Optimized 2-micron Optical Parametric Chirped Pulse Amplifier for High Harmonic Generation," XVI International Conference on Ultrafast Phenomena, Stresa, Italy, June 2008.

[4] S.-W. Huang, et al., "High-Power, Few-Cycle, Phase-stabilized 2.2- $\mu \mathrm{m}$ OPCPA," submitted to this conference. 